\title{
An Organic Radical Solid Solution with Strong Ferromagnetic Exchange
}

\author{
Hidenori Murata, Joel T. Mague, Safo Aboaku, Naoki Yoshioka, Paul M. Lahti*
}

\section{Supporting Material}

\section{Experimental details}

Page S1

Methodology for crystallographic analysis

Table S1. Summary of bonding parameters and contacts for F4BImNN/BImNN alloy crystal. $\quad$ S2

Figure S1. Mass spectrum (EI) of a crystal of F4BImNN/BImNN alloy. $\quad$ S4

Figure S2. HPLC trace of a crystal of F4BImNN/BImNN alloy. $\quad$ S5

Details of magnetic analysis $\quad$ S6

Figure S3. F4BImNN polycrystalline powder EPR, X-band, at varying temperatures. $\quad$ S7

Figure S4. F4BImNN/BImNN alloy polycrystalline powder EPR, X-band, at varying temperatures. S7

Figure S5. F4BImNN polycrystalline powder EPR average g-values from Figure S3. $\quad$ S8

Figure S6. F4BImNN/BImNN alloy polycrystalline powder EPR average g-values from $\quad$ S8

Figure S4.

Figure S7. F4BImNN, BImNN, and F4BImNN/BImNN alloy FTIR spectra. 


\section{Experimental details.}

F4BImNN was synthesized by the method of Murata et al.[1]

BImNN was synthesized by the method of Yoshioka et al.[2]

The alloy crystals of BImNN and F4BImNN were crystallized by the slow diffusion method using dichloromethane and methanol. BImNN and F4BImNN (1:1 molar ration) were dissolved in a small amount of dichloromethane in a open screw-top vial, which was placed in a larger vial containing methanol. The larger vial was stoppered, and the assemblage allowed to stand for several days. Deep blue, needle crystals were obtained.

\section{Methology for crystallographic analysis.}

A dark blue, needle crystal of the F4BImNN/BImNN alloy was cut to $0.25 \times 0.09 \times 0.03 \mathrm{~mm}$, affixed to a CryoLoop ${ }^{\circledR}$ with a drop of Paratone ${ }^{\circledR}$ oil and placed in the cold nitrogen stream of the Bruker Kryoflex low temperature accessory. Intensity data were collected on a Bruker-AXS Smart APEX CCD diffractometer at $100 \mathrm{~K}$ using three sets of 606 frames having a width of $0.3^{\circ}$ in $\omega$ and collected, respectively, at $\phi=0,120$ and $240^{\circ}$ for a total time of $90 \mathrm{sec} /$ frame. An initial orthorhombic unit cell and orientation matrix was obtained from approximately 900 reflections having $I / \sigma(I) \geq 20$ chosen from diverse regions of reciprocal space using the indexing and cell refinement routines of the SMART[3] software. The raw data were processed with the SAINT+ program[4] and, as there were few data at high angles because of the small crystal size, a resolution cutoff of $0.9 \AA^{-1}$ was imposed in order to improve the integration of the lower angle data. Empirical corrections for absorption and possible crystal decay were performed with SADABS[5] with averaging of symmetry equivalent reflections, including Friedel opposites.

The structure was solved by direct methods and refined by a full-matrix, least-squares process. As the refinement proceeded, it was readily evident that the displacement parameters for the fluorine atoms were significantly larger than those of the other atoms, notably those of the two oxygen atoms. This is consistent with analytical data indicating that the crystals studied are essentially a 1:1 mixture of F4BImNN and BImNN. The refinement was completed by modeling the molecule in the asymmetric unit with four fluorine and four hydrogen atoms, each of occupancy 0.5 on the periphery of the sixmembered ring. All hydrogen atoms attached to carbon were placed in calculated positions while that attached to $\mathrm{N} 2$ was placed in the

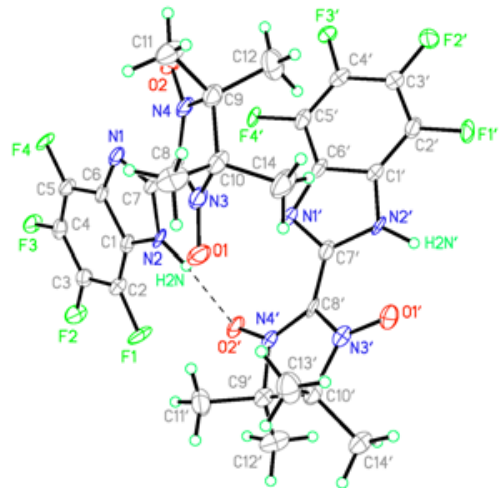
location provided by a difference map. All were included as riding contributions with isotropic displacement parameters 1.2-1.5 times those of the attached atoms. All computations associated with structure solution, refinement and presentation of results were performed with the SHELXTL[6] program package. Relevant crystal and refinement data are presented in Table 1 of the main text.

There is a significant hydrogen bond between $\mathrm{N} 2 \mathrm{H}$ and $\mathrm{O} 2$ in the neighboring molecule at $[x+1 / 2$, $y, 1 / 2-\mathrm{z}]$. The planes of the two five-membered rings are inclined at an angle of $16.6(4)^{\circ}$.

\section{References:}

1. Murata, H.; Delen, Z.; Lahti, P. M. Chem. Mater. 2006, 18, 2625-2627.

2. Yoshioka, N.; Irisawa, M.; Mochizuki, Y.; Kato, T.; Inoue, H.; Ohba, S. Chem. Lett. 1997, 251-252.

3. Bruker-AXS. 2000. SMART, Version 5.625, Madison, WI.

4. Bruker-AXS 2004. SAINT+, Version 7.03, Madison, WI

5. Sheldrick, G, M. 2002. SADABS, Version 2.05. University of Göttingen, Germany.

6. Bruker-AXS. 2000. SHELXTL, Version 6.10, Madison, WI. 
Table S1. Summary of bonding parameters and contacts for F4BImNN/BImNN alloy crystal.
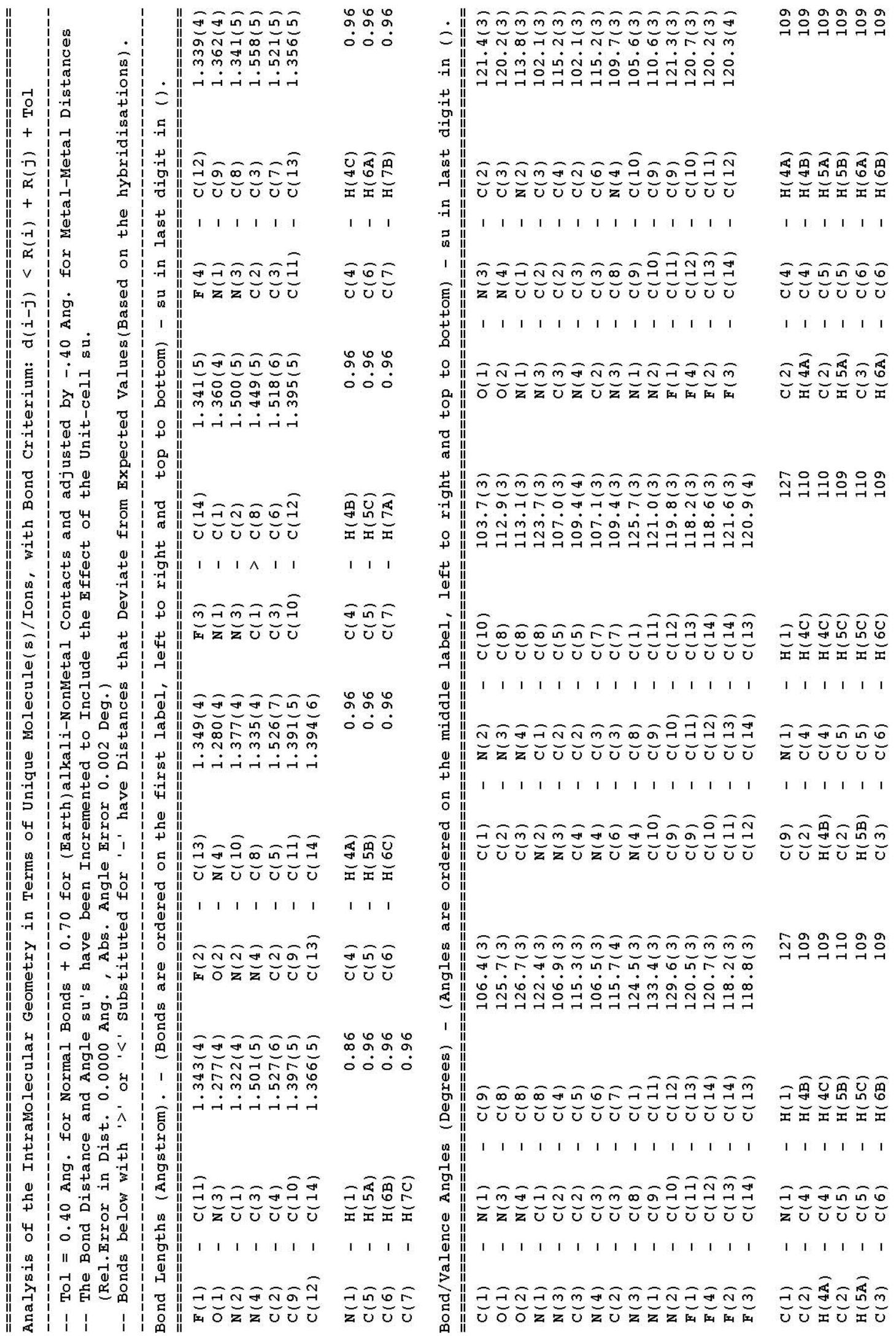
Table S1. Summary of bonding parameters and contacts for F4BImNN/BImNN aloy crystal, cont'd.

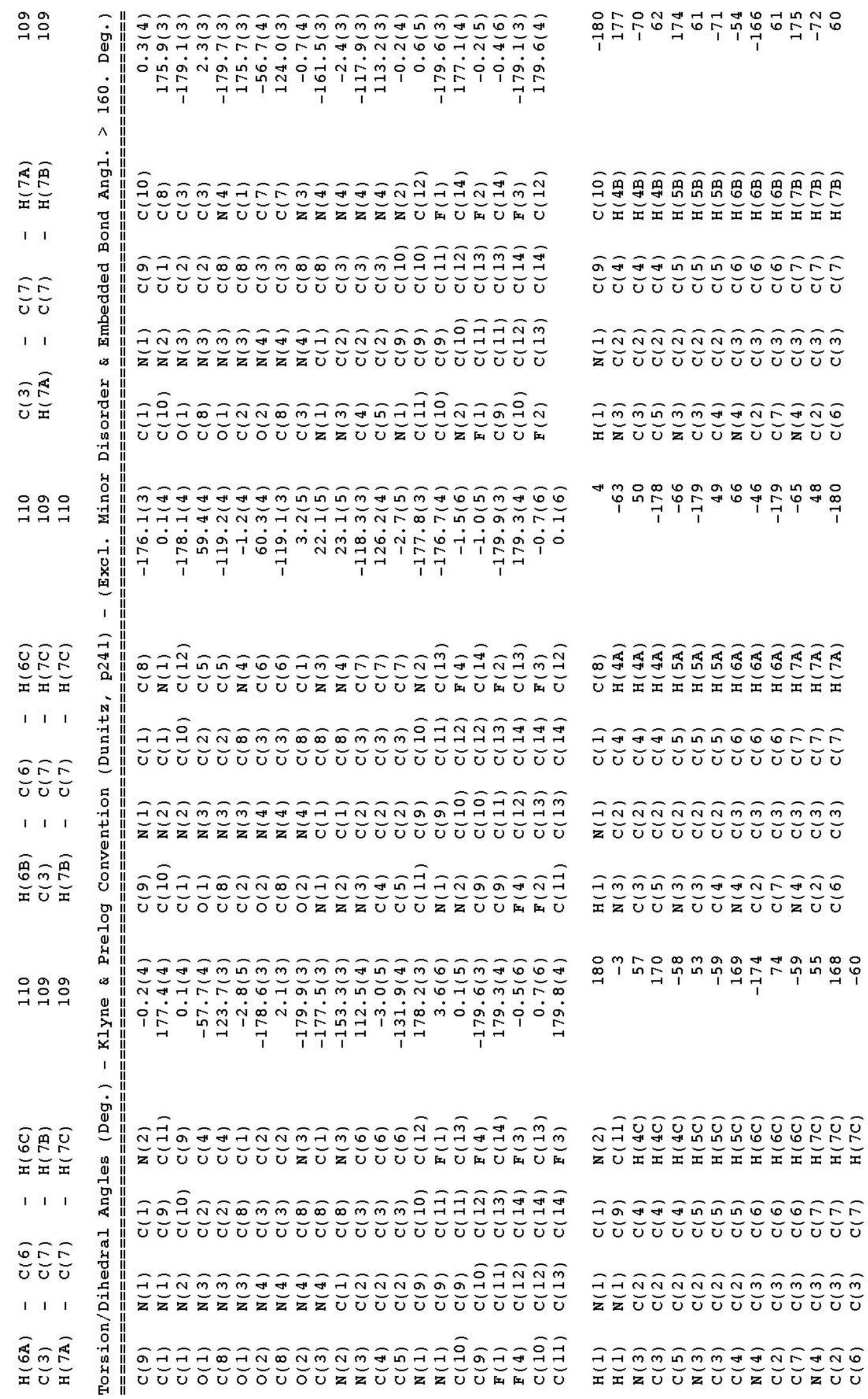


Figure S1. Mass spectrum (EI) of a crystal of F4BImNN/BImNN alloy.

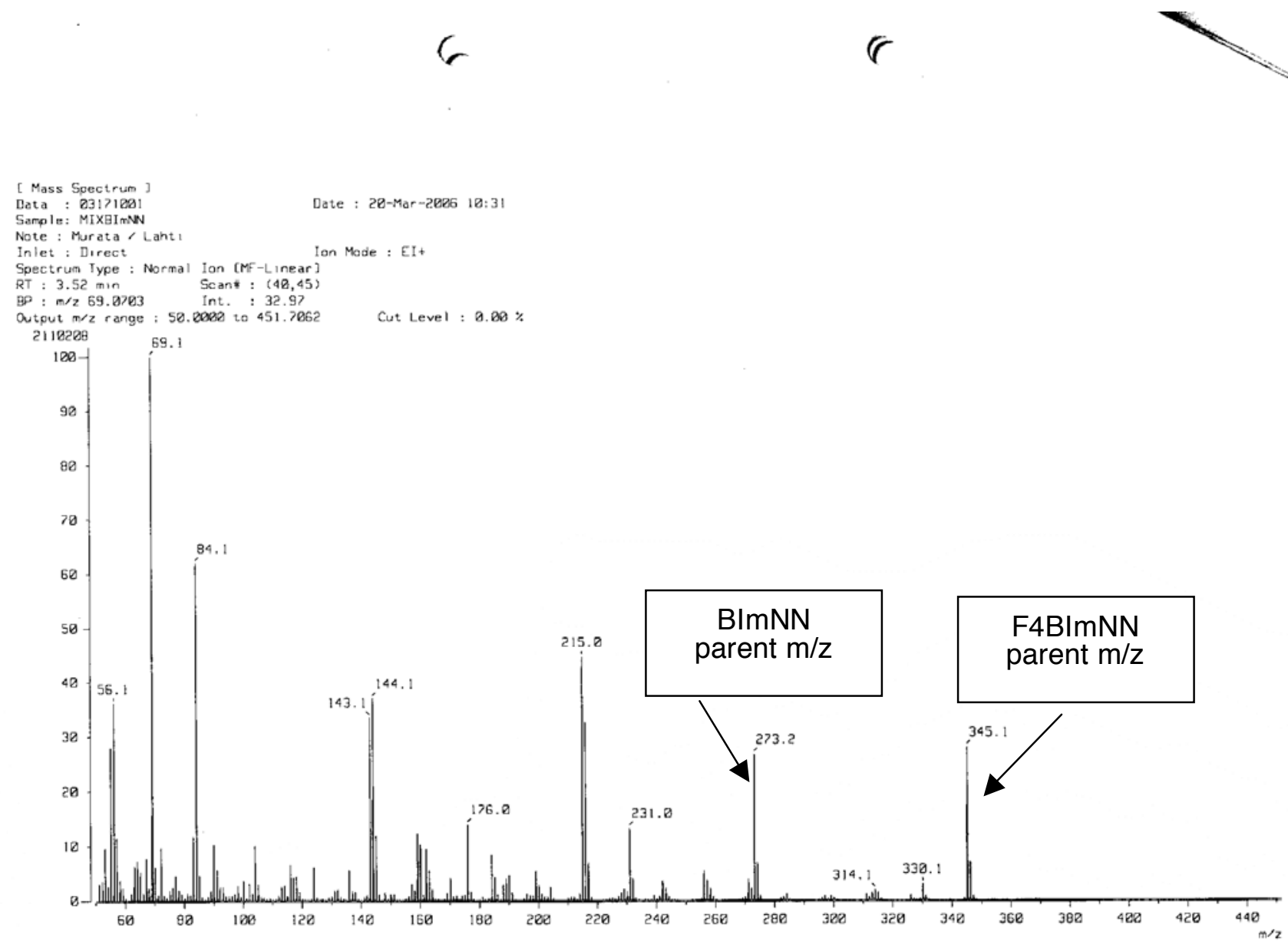


Figure S2. HPLC analysis of a crystal of F4BImNN/BImNN alloy.

$\underline{\text { Running Conditions: }}$

- Instrument: Waters Alliance 2695 HPLC with Waters 2487 dual wavelength absorbance detector

- Column: Waters C18, L = $150 \mathrm{~mm}$

- Mobile Phase: MeOH:Water (70:30 v:v)

- Temperature: Room Temperature

- Flow Rate: $0.5 \mathrm{~mL} / \mathrm{min}$

- Injection volume: 20 microL

- Detection Wavelength: $300 \mathrm{~nm}$

- $\quad$ Run Time: $10 \mathrm{~min}$

- Blank: pure $\mathrm{MeOH}$

All samples are dissolved in $\mathrm{MeOH}$. Each sample injection was followed by a blank injection. The detector response ratio for $\mathrm{mol} / \mathrm{mol}$ F4BImNN:BImNN was established to be 0.737 by control experiments using known ratios of F4BImNN/BImNN. The integral ratio F4BImNN/BImNN below is 0.640 , so the sample molar ratio F4BImNN/BImNN $=0.868$.

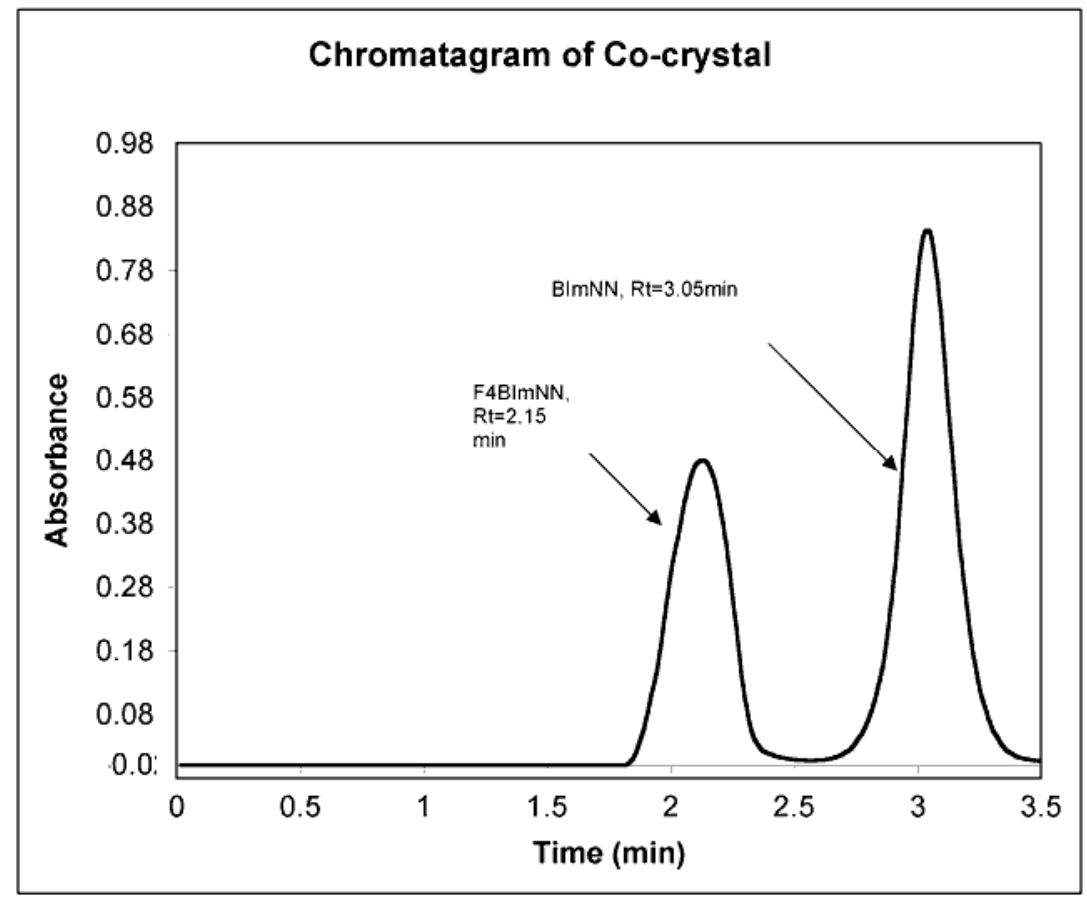




\section{Details of magnetic analysis}

(1) Magnetic measurements were carried out on crushed, polycrystalline samples of F4BImNN/BImNN alloy placed into gelatin capsules and held in place with a small amount of cotton. The samples were placed in a plastic straw and inserted into a Quantum Design MPMS-5 SQUID magnetometer. Samples were measured at various applied fields $(\mathrm{H})$ between 1.8-300 K. Magnetic moments M were converted to molar moments using the molecular weight of the alloy determined from the HPLC mixture ratio (see previous information), then to molar magnetic susceptibilities using $\chi=M / H$.

(2) The equation shown below [Swank, D. D.; Landee, C. P., Willet, R. D. Phys. Rev. B, 1979, 20, 2154] was fitted by a nonlinear least squares procedure to the $\chi T(T)$ data, where $T I C$ is a temperature independent correction factor that includes contributions from the sample holder and the sample diamagnetism. Constants used were $\beta=$ Bohr magneton, $\mathrm{k}=$ Boltzmann constant, $\mathrm{N}=$ Avogadro's number, $\mathrm{T}=\mathrm{Kelvin}$ temperature. The Landé factor $\mathrm{g}$ was fixed at $\mathrm{g}=2.006$ based on the ESR spectrum of the mixture at toom temperature. The exchange constant $\mathbf{J}$, the mean field constant $\boldsymbol{\theta}$, and TIC were allowed to vary in the least squares analysis, using the program PSI-Plot ${ }^{\mathrm{TM}}$ for Windows, version 8 by PolySoftware International Inc. of Pearl River NY. The data and fits in the main article are plotted post TIC-correction, so the article shows corrected $\chi$.

$$
\begin{gathered}
\{\chi-T I C\} \cdot T=\frac{N g^{2} \beta^{2} T}{4 k(T-\theta)} \cdot\left[\frac{1+A \cdot Q+B \cdot Q^{2}+C \cdot Q^{3}+D \cdot Q^{4}+E \cdot Q^{5}}{1+F \cdot Q+G \cdot Q^{2}+H \cdot Q^{3}+I \cdot Q^{4}}\right]_{\text {A }=2.7979916, \mathrm{G}=7.0086780, \mathrm{H}=8.65438644, \mathrm{I}=4.5743114 ;}^{\mathrm{A}=5.7979916, \mathrm{~B}=16.902653, \mathrm{C}=29.376885, \mathrm{D}=29.832959, \mathrm{E}=14.036918,} \\
\mathrm{Q}=\mathrm{J} /(2 \mathrm{kT})
\end{gathered}
$$

This equation is described by Swank et al. (above) as deriving from the Pade-based model of Baker, G. A., Jr.; Rushbrooke, G. S.; Gilbert, H. E. Phys. Rev., 1964, 135, A1272, who use the spin Hamiltonian.

$$
H=-2 J \sum S_{i} \cdot S_{j}
$$

We thank Prof. C. Landee for helpful discussions of their paper (Swank et al., cited at the top of this page). 
Figure S3. F4BImNN polycrystalline powder EPR, X-band, at varying temperatures: $100 \mathrm{kHz}$ modulation frequency, 3.0 G modulation amplitude, $0.2 \mathrm{~mW}$ microwave power, $9.37 \mathrm{GHz}$.

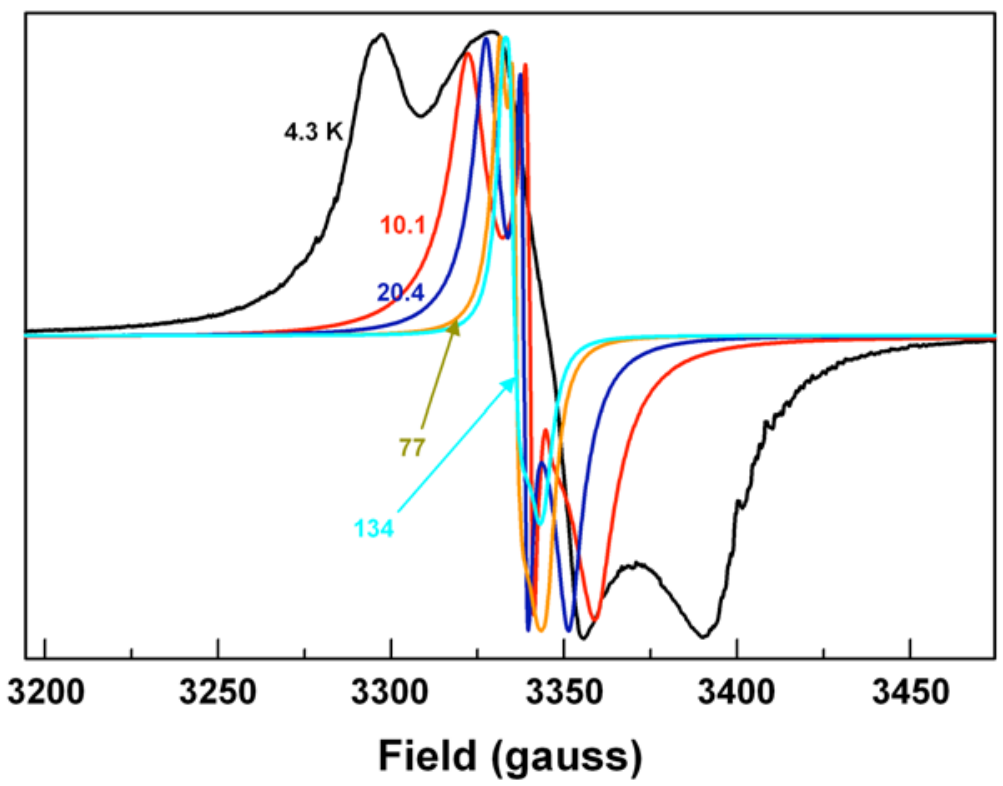

Figure S4. F4BImNN/BImNN alloy polycrystalline powder EPR spectra, X-band, at varying temperatures: $100 \mathrm{kHz}$ modulation frequency, $1.0 \mathrm{G}$ modulation amplitude, $0.2 \mathrm{~mW}$ microwave power, $9.37 \mathrm{GHz}$.

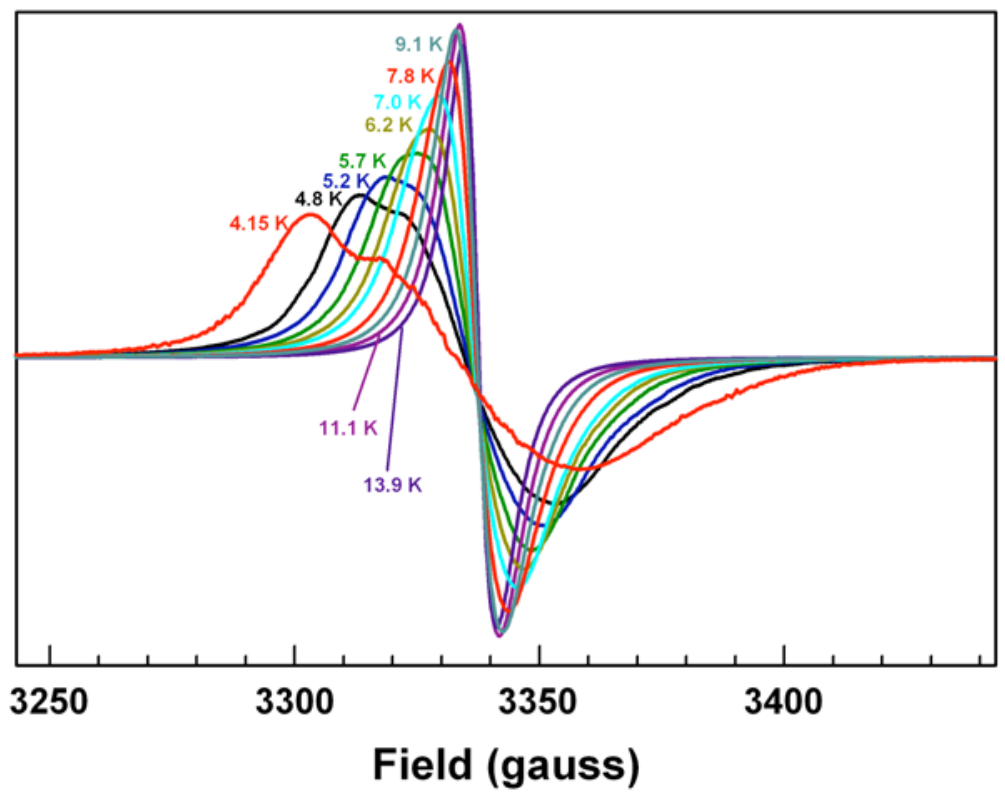

For each experiment set above, a single crystal was crushed in a $4 \mathrm{~mm}$ O.D. quartz ESR tube, using a quartz rod. The sample tube was tapped several times to agitate the powder. 
Figure S5. F4BImNN polycrystalline powder EPR average g-values from Figure S3.

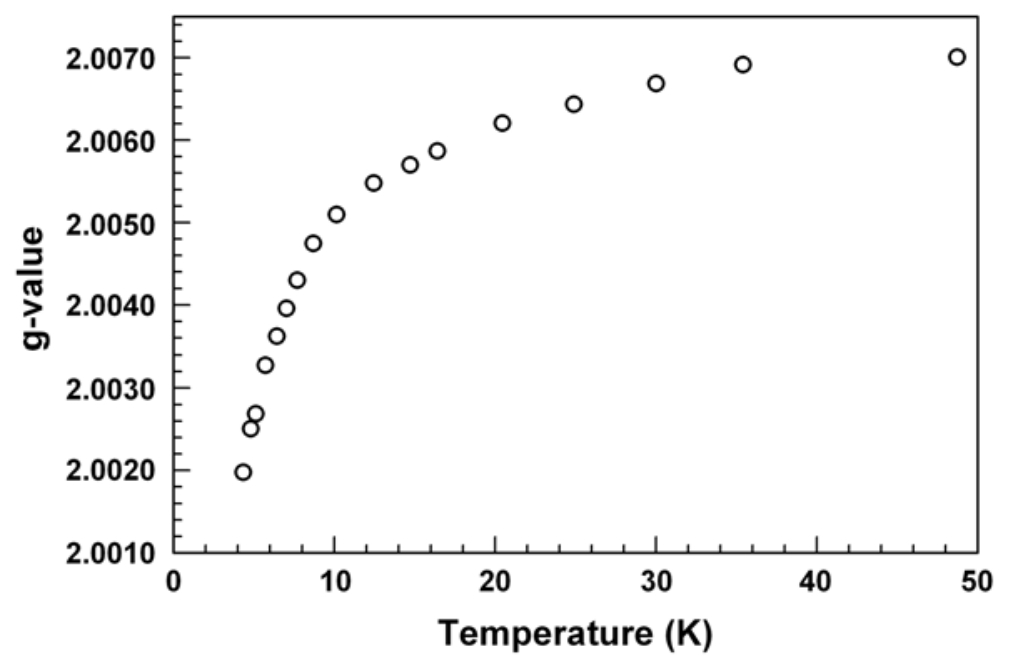

Figure S6. F4BImNN/BImNN alloy polycrystalline powder EPR average g-values from Figure S4.

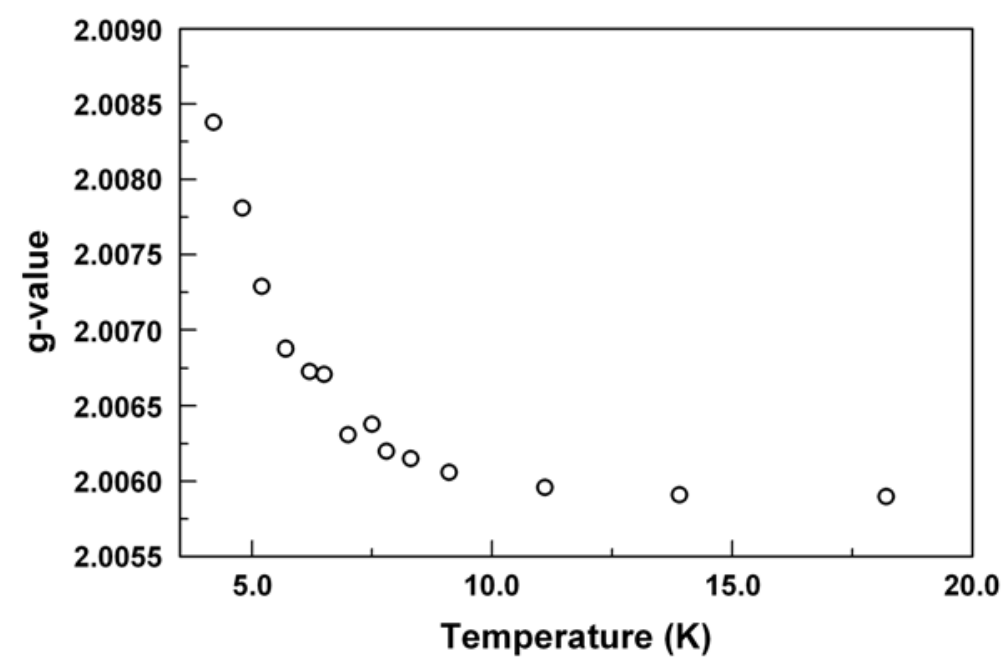


Figure S7. F4BImNN, BImNN, and F4BImNN/BImNN alloy FTIR spectra. All spectra were obtained as pressed $\mathrm{KBr}$ pellets.
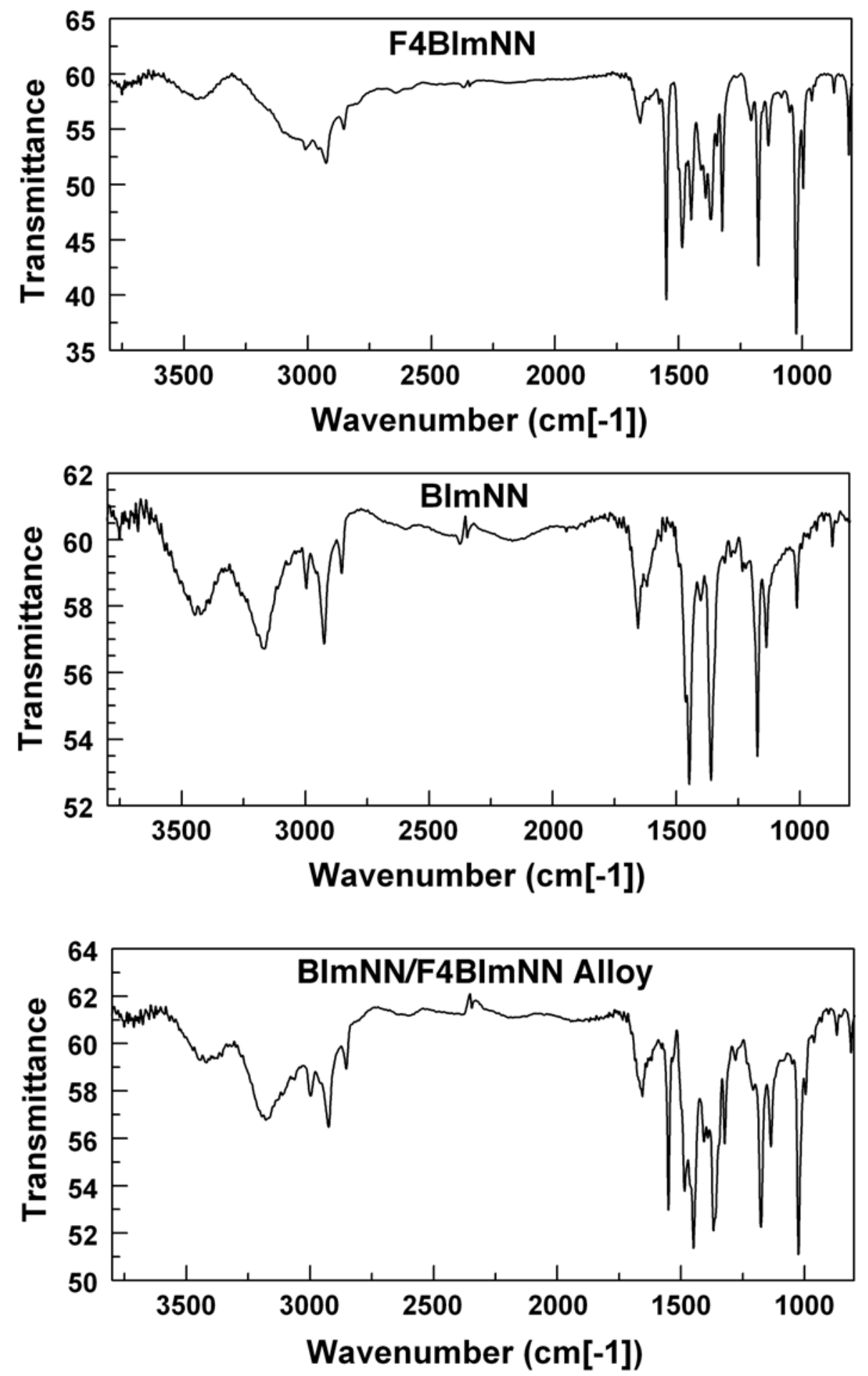